\title{
Desmoplastic Reaction and Tumor Budding in Cervical Squamous Cell Carcinoma are Prognostic Factors for Distant Metastasis: A Retrospective Study
}

This article was published in the following Dove Press journal: Cancer Management and Research

\section{Lanqing Cao \\ Ping-Li Sun (D) \\ Yangyang $\mathrm{He}$ \\ Min Yao (D) \\ Hongwen Gao}

Department of Pathology, The Second Hospital of Jilin University, Changchun, jilin I3004I, People's Republic of China
Purpose: An accurate risk assessment system for disease metastasis or recurrence could improve the cancer management practice in cervical squamous cell carcinoma (CxSCC) patients, which has few definite prognostic predictors. Previous studies have indicated the important utility of stromal features in determining cancer biological behavior; however, it lacks histopathologic or morphologic criteria for its evaluation. Therefore, this present study aimed to comprehensively catalog histopathological features of mesenchymal stroma to determine the prognostic value of these features in CxSCC.

Patients and methods: We histologically and immunohistochemically evaluated the stromal features in the primary tumors of $122 \mathrm{CxSCC}$ patients. The follow-up duration was 41.25 months (range: 3-80.77 months). Multivariate proportional hazard regression models were used to identify the top classifier for distant metastasis-free survival (DMFS) prediction.

Results: Lymph-vascular invasion (LVI), lymph node metastasis (LNM), tumor-nodemetastasis (TNM) stage and tumor budding were positively correlated with distant metastasis ( $P<0.001, P<0.001, P<0.001$ and $P=0.012$, respectively). Distant metastasis was also associated with the immature desmoplastic reaction $(\mathrm{DR})(P=0.002)$, high level of cancerassociated fibroblasts ( $P=0.003$ ), vasohibin-1 (VASH1)-positive microvessels $(P=0.027)$, and the VASH1/CD31 ratio $(P=0.004)$. Multivariate COX proportional hazard regression models revealed that LVI, LNM, and DR were independent predictors of poor DMFS in CXSCC patients. Conclusion: Primary tumor histologic stromal features, especially DR, may be useful in predicting distant metastasis in patients with $\mathrm{CxSCC}$.

Keywords: stromal features, squamous cell carcinoma, distant metastasis, desmoplastic reaction

\section{Introduction}

Metastasis is one of the major causes of therapy failure and subsequent death in patients with cervical squamous cell carcinoma (CxSCC). Moreover, lymphatic vessel invasion usually indicates metastasis and poor prognosis. ${ }^{1,2}$ Recent data have revealed that tumor-associated microenvironment is a vital determinant for the susceptibility to human papillomavirus (HPV)-related CxSCC. ${ }^{3}$ Tumor microenvironment factors, including the presence of cancer-associated fibroblasts (CAFs), microvessel density (MVD), vasohibin-1 (VASH1) and nestin expression in newly formed stromal vessels, have been identified as reliable biomarkers of prognostic predictors in various human malignancies, including $\mathrm{CxSCC}^{4-7}$

Tumor cells require a specific microenvironment to survive and grow, and emerging data have revealed that fibroblasts could alter cancer behavior by releasing growth
Correspondence: Min Yao Department of Pathology, The Second Hospital of Jilin University, 218 Ziqiang Road, Changchun, Jilin I3004I, People's Republic of China

Tel/Fax +86-43I-8I I36933

Email yaomin@jlu.edu.cn 
factors and cytokines and the dynamic extracellular matrix (ECM), which also favors tumor progression. ${ }^{8}$ ECM is upregulated in tumor stroma and creates the deposition of a reactive stroma; this is known as a desmoplastic reaction (DR). ${ }^{9}$ In recent studies, DR has been demonstrated as a successful biomarker that was shown to stratify postoperative survival. ${ }^{10,11}$ Because of the urgent need of the top biomarkers which may have the potential to enable early diagnosis, predict patient's prognosis, and facilitate the prediction of disease recurrence or metastasis.

Our study tried to investigate the prognostic value of stroma features in CxSCC. Due to the large number of histological features and small patient cohort sizes, we aimed to identify prognostic predictors that are crucial for the assessment of metastasis in CxSCC patients.

\section{Materials and Methods}

\section{Patients and Tissue Specimens}

In the present study, we collected the specimens and clinicopathology data of 122 non-keratinizing $\mathrm{CxSCC}$ patients who underwent radical hysterectomy and pelvic lymphadenectomy at the Second Hospital of Jilin University between January 2009 and January 2013. Patients who received preoperative neoadjuvant therapy were excluded from our study. The last follow-up date was May 2018 and the mean follow-up duration is 41.25 months (range: $3-80.77$ months). The end point of distant metastasis-free survival (DMFS) was calculated from the date of diagnosis of the primary tumor to the first distant metastasis occurrence. Distant metastases included the intraperitoneal disease and inguinal lymph nodes; it excluded metastases to the vagina, adnexa and pelvic serosa. This study was approved by the Ethics Committee of The Second Hospital of Jilin University (Jilin, China) (IRB approval number: 2019092). The TNM stages were assessed using the American Joint Committee on Cancer's Cancer Staging Manual, 8th edition.

\section{Immunohistochemistry}

For studying protein expression in $\mathrm{CxSCC}$, paraffinembedded tissue sections were deparaffinized and incubated with antibodies against nestin (ZA-0628), CD31 (ZM-0044), CD34 (ZM-0046), smooth muscle actin (SMA) (ZM-0003) (Zhongshan Golden Bridge Biotechnology LLC, Beijing, China), and VASH1 (Cat. \#ab219790; Abcam, Cambridge, MA, USA; 1:50 dilution in PBS) and then with biotinconjugated secondary antibody PV-9001 IHC kit (Zhongshan Golden Bridge Biotechnology LLC, Beijing,
China) at $37^{\circ} \mathrm{C}$ for $30 \mathrm{~min}$. The color reaction was performed using a 3,3'-diaminobenzidine kit (Zhongshan Golden Bridge Biotechnology LLC).

\section{DR Categorization}

Hematoxylin and eosin (H\&E) staining slides were used as routine pathological procedures and viewed under a light microscope at a low magnification to evaluate the myxoid stroma and "keloid-like" collagen at the tumor stroma. A median number of 2 (range, 1-10) glass slides (per case) were examined in this cohort.

Thick bundles of collagen with eosinophilic hyalinization were identified as "keloid-like" collagen. ${ }^{12}$ An amorphous stromal substance that comprises an amphophilic or basophilic ECM is defined as myxoid stroma. ${ }^{12}$ Based on the presence of keloid-like collagen or myxoid stroma, DR was histologically categorized as mature, intermediate, or immature, ${ }^{11}$ Immature DR was classified when stroma was observed with myxoid changes. In the ones with no myxoid stroma, the intermediate DR was identified when the "keloid-like" collagen was mixed with the mature stroma. Finally, the mature DR was classified when the stroma did not contain myxoid stroma or "keloidlike" collagen but typically contained mature collagen fibers. ${ }^{11}$ Two pathologists (MY and LQC) evaluated all the glass slides of the whole tumor to determine the DR pattern for each case. Previously reported the $\kappa$ coefficient for intraobserver and interobserver variations in DR categorization was $0.79^{13}$ and $0.69,{ }^{14}$ respectively.

\section{Microvessel Density, CAFs, and Tumor Budding}

Tissue sections were visualized under low magnification $(40 \times$ and $100 \times)$, and intratumoral microvessels were evaluated based on the vessel architecture (i.e., when the vessel architecture was lined with endothelial cells that were positively stained with anti-CD31 and anti-VASH1). The areas of the highest microvessels or "hot spots" were detected. ${ }^{4,6}$ Every slide was evaluated at three different hot-spot locations, and counted at $200 \times$ magnification.

The frequency of CAFs was evaluated by examining the entire tumor stroma and was quantitatively assessed using a five-scale scoring system as previous study. ${ }^{15}$ For statistical analysis, the cases with low scores were compared to the cases with high scores. ${ }^{15}$

Tumor budding was defined as a small dissociation of tumor cell complexes that consisted of $<5$ tumor cells in the peritumoral stroma. In recent studies, ${ }^{16-18}$ tumor budding 
was detected throughout the whole tumor section and scored within the greatest budding activity. In the current study, tumor budding was classified low as $1-14$ and high as $\geq 15$ budding in 10 high-power fields (HPFs), respectively.

\section{Statistical Analysis}

All statistical analyses were performed using SPSS statistical software, version 21.0 (SPSS Inc., Chicago, IL, USA). Continuous variables were evaluated using the Mann-Whitney $U$-test, or the chi-square test was used to analyze categorical variables.

Further, significant variables $(P<0.1)$ from the univariate analyses were included in the multivariate analyses. A multivariate Cox proportional hazards model with the stepwise Wald method was used to obtain hazard ratios for distant metastasis and to identify factors that affect DMFS. Kaplan-Meier survival curves and Log-rank tests were used to evaluate DMFS. For all analyses, $P<0.05$ was considered statistically significant. Analyses of interobserver variance were performed using Cohen's Kappa algorithm. As only planned hypothesis testing was performed, no corrections for multiple testing were necessary for this study. ${ }^{19}$

\section{Results}

\section{Patient Characteristics}

The median age of the patients included in this study was 50 years-old (range: 26-76 years). Clinicopathological data of each patient, such as age at diagnosis and tumor characteristics, including TNM stage, lymph-vascular invasion (LVI), and lymph node metastases (LNM), were obtained. Follow-up data were recorded for all 122 patients. All patients received followed-up care for a mean period of 41.25 months, and the last follow-up date was May 2018. Twenty of 122 CxSCC patients (16.4\%) had distant metastases which included 12 lung metastases, 6 supraclavicular lymph node metastases, and 2 bone metastases.

\section{Associations Between Distant Metastasis and Clinicopathological Parameters}

Table 1 shows the association between clinicopathological parameters and distant-metastasis status, and tumor budding is shown in Figure 1. Overall, our results demonstrated that distant metastasis was positively correlated with TNM stage, LVI, LNM, and tumor budding $(P<0.001, P<0.001$, $P<0.001$ and $P=0.012$, respectively).
Table I Univariate Analysis Results for Clinicopathological Parameters for Predicting Distant Metastasis

\begin{tabular}{|c|c|c|c|c|c|}
\hline \multirow[t]{2}{*}{ Characteristics } & \multirow{2}{*}{$\begin{array}{l}\text { All } \\
\text { Patients }\end{array}$} & \multicolumn{3}{|l|}{ DM } & \multirow[t]{2}{*}{$P$ value } \\
\hline & & \multicolumn{2}{|c|}{ Absent No. (\%) } & $\begin{array}{l}\text { Present } \\
\text { No. (\%) }\end{array}$ & \\
\hline Age & $\begin{array}{l}50.38 \pm \\
6.8\end{array}$ & \multicolumn{2}{|c|}{$49.71 \pm 6.9$} & $50.38 \pm 6.4$ & 0.773 \\
\hline TNM stage & $\begin{array}{l}\text { I } \\
\text { II } \\
\text { III }\end{array}$ & $\begin{array}{l}83 \\
9 \\
30\end{array}$ & $\begin{array}{l}78(94.0) \\
9(100) \\
14(46.7)\end{array}$ & $\begin{array}{l}5(6.0) \\
0(0.0) \\
16(53.3)\end{array}$ & $<0.001$ \\
\hline T stage & $\begin{array}{l}\text { Tla } \\
\text { Tlb } \\
\text { T2 }\end{array}$ & $\begin{array}{l}28 \\
82 \\
12\end{array}$ & $\begin{array}{l}24(85.7) \\
68(82.9) \\
9(75.0)\end{array}$ & $\begin{array}{l}4(14.3) \\
14(17.1) \\
3(25.0)\end{array}$ & 0.712 \\
\hline $\begin{array}{l}\text { Lymph-node } \\
\text { status }\end{array}$ & $\begin{array}{l}\text { No } \\
\text { NI }\end{array}$ & $\begin{array}{l}92 \\
30\end{array}$ & $\begin{array}{l}87(94.6) \\
14(46.7)\end{array}$ & $\begin{array}{l}5(5.4) \\
16(53.3)\end{array}$ & $<0.001$ \\
\hline LVI & $\begin{array}{l}\text { Absent } \\
\text { Present }\end{array}$ & $\begin{array}{l}105 \\
17\end{array}$ & $\begin{array}{l}98(93.3) \\
3(17.6)\end{array}$ & $\begin{array}{l}7(6.7) \\
14(82.4)\end{array}$ & $<0.001$ \\
\hline $\begin{array}{l}\text { Tumor budding } \\
\text { (10 HPFs) }\end{array}$ & $\begin{array}{l}0 \\
1-15 \\
>15\end{array}$ & $\begin{array}{l}29 \\
65 \\
28\end{array}$ & $\begin{array}{l}26(89.7) \\
57(87.7) \\
18(64.3)\end{array}$ & $\begin{array}{l}3(10.3) \\
8(12.3) \\
10(35.7)\end{array}$ & 0.012 \\
\hline
\end{tabular}

Note: Statistical analysis was conducted by Mann-Whitney U-test and Fisher exact test. Abbreviations: TNM, tumor-node-metastasis; T, tumor; LVI, lymph-vascular invasion; HPFs, high-power fields DM, distant metastasis.

\section{Associations Between Stromal Features and Distant Metastasis}

The associations between distant metastasis and stromal features are summarized in Table 2, and an example of DR is presented in Figure 1. Our results revealed that there was a statistically significant relationship between distant metastasis and DR. Specifically, immature stroma was more common in tumors of distant-metastasis group (8 of $19 ; 42.1 \%$ ), while mature and intermediate stroma were more predominant in tumors without distant metastasis. Further, the number of VASH1-positive microvessels, the VASH1/CD31 ratio, and CAFs levels were significantly higher in distant-metastasis group than those in without distant-metastasis group $(P=0.027, P=0.004$, $P=0.003$, respectively) (Figure 2).

\section{Association Between Stromal Features in CxSCC and Patient Survival}

Kaplan-Meier curves and Log-rank test were used to analyze DMFS rates of the CxSCC patients after they were stratified according to stromal features and clinicopathological parameters (Table 3) (Figure 3). Findings from this analysis demonstrated that there was a significant relationship between distant metastasis and 

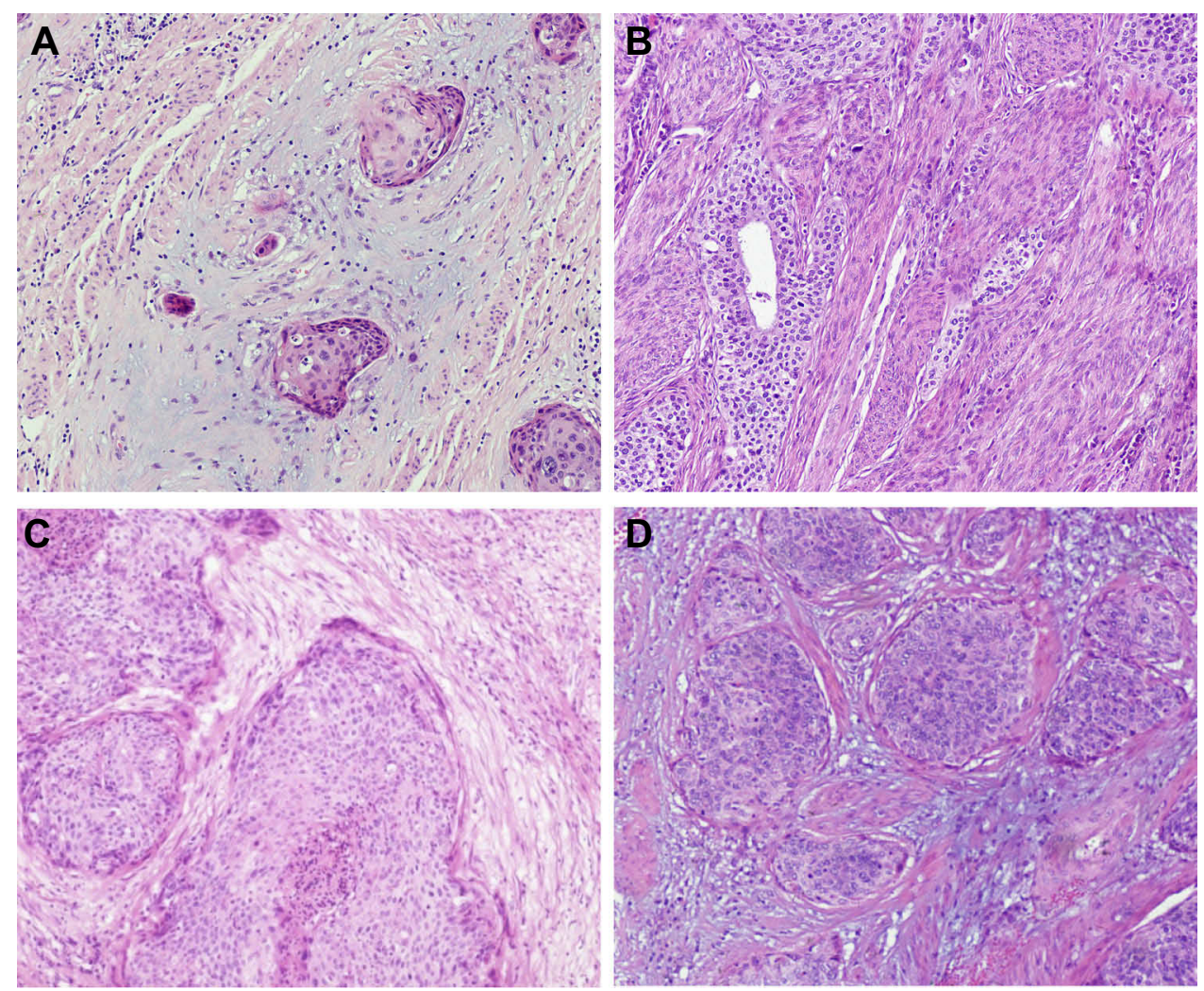

Figure I Morphological assessment tumor budding and desmoplastic reaction of the primary tumor (H\&E staining). (A) a CxSCC with high tumor budding activity in the myxoid stroma; (B) a CxSCC with mature DR; (C) a CxSCC with intermediate; (D) a CxSCC with immature (original magnification $\times 200$ ).

TNM stage, LVI, LNM, tumor budding, DR, CAFs, 95\% CI: 1.081-5.173) were independent determinants of VASH1-positive microvessels, and VASH1/CD31. patient survival in the multivariate Cox regression model. Additionally, the presence of LVI (hazard ratio [HR], 6.187; 95\% confidence interval [CI]: 2.042-21.796),

\section{Discussion}

LNM (HR, 5.770; 95\% CI: 1.427-23.395), tumor budding

In this study, we analyzed the impact of prognostic pre(HR, 2.782; 95\% CI: 1.372-5.891), and DR (HR, 2.363; dictors on metastasis and survival in patients with $\mathrm{CxSCC}$.

Table 2 Univariate Analysis Results for Stromal Features for Predicting Distant Metastasis

\begin{tabular}{|c|c|c|c|c|c|}
\hline \multicolumn{2}{|c|}{ Characteristics } & \multirow{3}{*}{$\begin{array}{l}\text { All Patients } \\
98 \\
5 \\
19\end{array}$} & \multicolumn{2}{|l|}{ DM } & \multirow{3}{*}{$\begin{array}{l}P \text { value } \\
0.002\end{array}$} \\
\hline & & & \multirow{2}{*}{$\begin{array}{l}\text { Absent No. (\%) } \\
87(88.8) \\
3(60.0) \\
11(57.9)\end{array}$} & \multirow{2}{*}{$\begin{array}{l}\text { Present No. (\%) } \\
\text { II (II.2) } \\
2(40.0) \\
8(42.1)\end{array}$} & \\
\hline DR & $\begin{array}{l}\text { Mature } \\
\text { Intermediate } \\
\text { Immature }\end{array}$ & & & & \\
\hline CAF & $\begin{array}{l}\text { High } \\
\text { Low }\end{array}$ & $\begin{array}{l}65 \\
57\end{array}$ & $\begin{array}{l}60(92.3) \\
41(71.9)\end{array}$ & $\begin{array}{l}5(7.7) \\
16(28.1)\end{array}$ & 0.003 \\
\hline VASHI status & Mean \pm SE & $15.34 \pm 0.81$ & $14.20 \pm 0.72$ & $|5.6| \pm 0.92$ & 0.027 \\
\hline Nestin status & Mean \pm SE & $14.63 \pm 1.72$ & $14.91 \pm 1.93$ & $14.48 \pm 1.01$ & 0.483 \\
\hline CD3I status & Mean \pm SE & $25.11 \pm 0.97$ & $26.06 \pm 1.03$ & $23.40 \pm 0.87$ & 0.131 \\
\hline VASHI/CD3I & Mean \pm SE & $0.61 \pm 0.01$ & $0.56 \pm 0.01$ & $0.66 \pm 0.01$ & 0.004 \\
\hline Nestin/CD3I & Mean \pm SE & $0.66 \pm 0.06$ & $0.68 \pm 0.06$ & $0.62 \pm 0.07$ & 0.761 \\
\hline Nestin/VASHI & Mean \pm SE & $0.92 \pm 0.32$ & $0.96 \pm 0.35$ & $0.87 \pm 0.27$ & 0.848 \\
\hline
\end{tabular}

Note: Statistical analysis was conducted by Mann-Whitney $U$-test and Fisher exact test.

Abbreviations: DR, desmoplastic reaction; CAF, cancer-associated fibroblasts; VASHI, vasohibin-I; SE, standard error; DM, distant metastasis. 

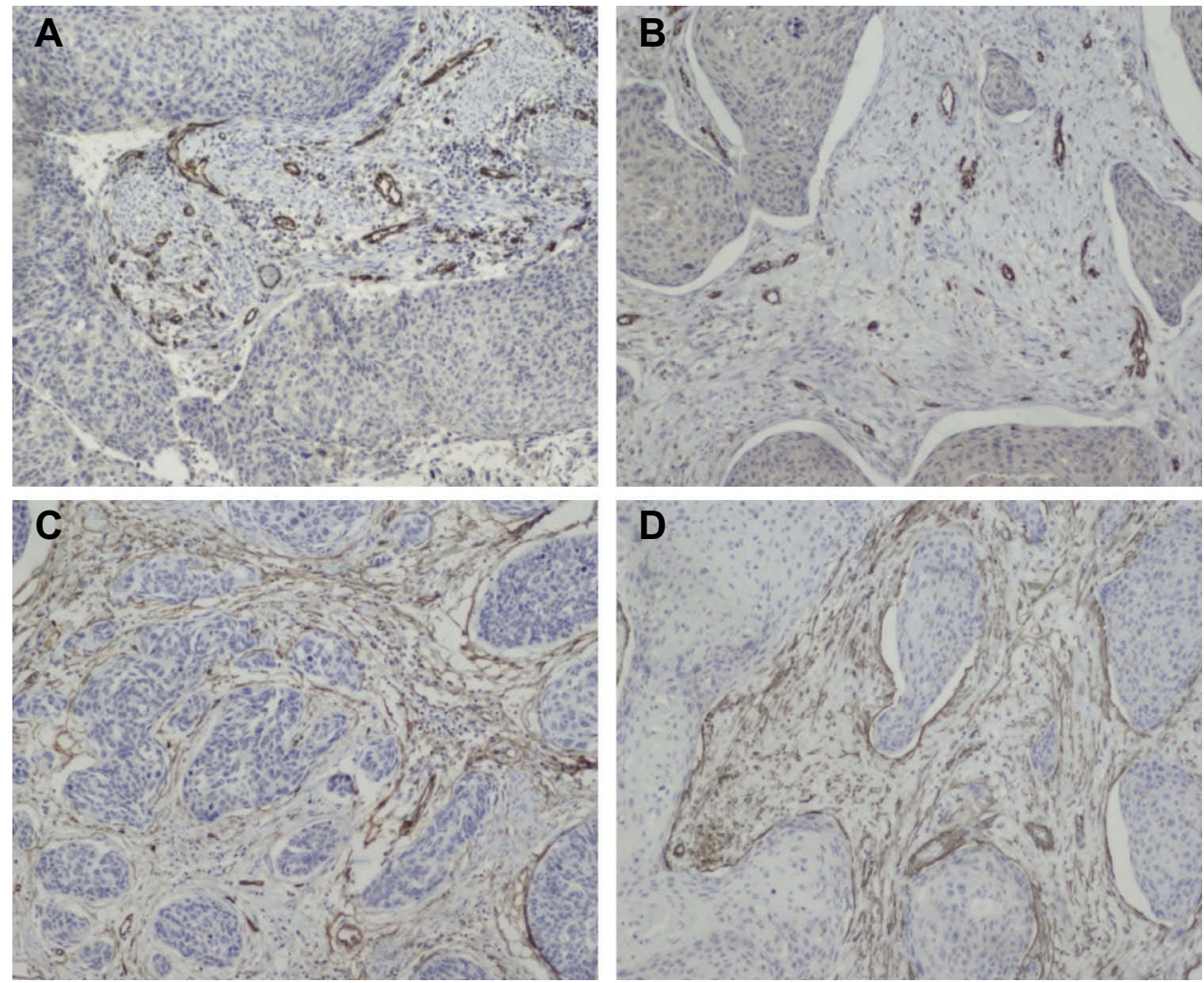

Figure 2 Immunohistochemical detection of the stroma features in cervical squamous cell carcinoma tissues. (A, B) CD3I-positive and VASHI-positive vessels in the intratumoral stroma; (C, D) CD34-positive and SMA-positive fibroblasts in tumor-adjacent stroma (original magnification $\times 200$ ).

Our results demonstrated that immature stroma, MVD, and CAFs in CxSCC were directly correlated with aggressive features; LVI, LNM, TNM stage, DR, VASH1-positive microvessels, the VASH1/CD31 ratio, and CAFs were significantly correlated with DMFS in patients with CxSCC; LVI, TNM stage, LNM, and DR were independent predictors of poor DMFS in this cohort.

Additionally, our data revealed that DR categorization could stratify the risk of recurrence in this cohort of patients. Specifically, the accumulative distant metastasis rate at 5 years postoperatively ranged from $11.2 \%$ to $42.1 \%$. The patients with mature stroma have favorable prognosis, while the immature stroma have most unfavorable prognosis. These results confirmed with previous studies that evaluated cohorts of patients with $\mathrm{CxSCC}$ at different stages. ${ }^{20}$ Our results suggest that the prognostic power of DR categorization may be independent from the anatomical extent of the disease. ${ }^{21,22}$ Notably, DR

Table 3 Univariate and Multivariate Analyses of Distant Metastasis-Free Survival for Patients with Cervical Squamous Carcinoma

\begin{tabular}{|c|c|c|c|c|c|c|c|c|}
\hline & \multicolumn{4}{|c|}{ DMFS } & \multicolumn{4}{|c|}{ DMFS } \\
\hline & \multirow{2}{*}{$\frac{\mathbf{H R}}{2.900}$} & \multicolumn{2}{|c|}{$95 \% \mathrm{Cl}$} & \multirow{2}{*}{$\begin{array}{l}P \text { value } \\
<0.00 \text { I }\end{array}$} & \multirow{2}{*}{$\begin{array}{l}\text { HR } \\
6.187\end{array}$} & \multicolumn{2}{|c|}{$95 \% \mathrm{Cl}$} & \multirow{2}{*}{$\begin{array}{l}P \text { value } \\
0.001\end{array}$} \\
\hline LVI & & 2.109 & 5.589 & & & 2.042 & 21.796 & \\
\hline LNM & 4.900 & 3.302 & 6.498 & $<0.001$ & 5.770 & 1.427 & 23.395 & 0.001 \\
\hline TNM stage & 3.453 & 2.452 & 6.789 & $<0.001$ & - & - & - & - \\
\hline Tumor budding & 6.923 & 5.469 & 7.481 & 0.005 & 2.782 & 1.372 & 5.891 & 0.007 \\
\hline DR & 5.492 & 4.723 & 6.876 & 0.002 & 2.363 & 1.081 & 5.173 & 0.027 \\
\hline CAF & 7.629 & 6.469 & 7.378 & 0.006 & - & - & - & - \\
\hline VASHI & 6.259 & 6.319 & 7.338 & 0.015 & - & - & - & - \\
\hline VASHI/CD3I & 6.529 & 5.451 & 7.368 & 0.011 & - & - & - & - \\
\hline
\end{tabular}

Notes: Statistical analysis was conducted by Log-rank tests and Cox proportional hazards model.

Abbreviations: LVI, lymph-vascular invasion; LNM, lymph node metastasis; TNM, tumor-node-metastasis; DR, desmoplastic reaction; CAF, cancer-associated fibroblasts; VASHI, vasohibin-I; Cl, confidence interval; HR, hazard ratio; DMFS, distant metastasis-free survival. 

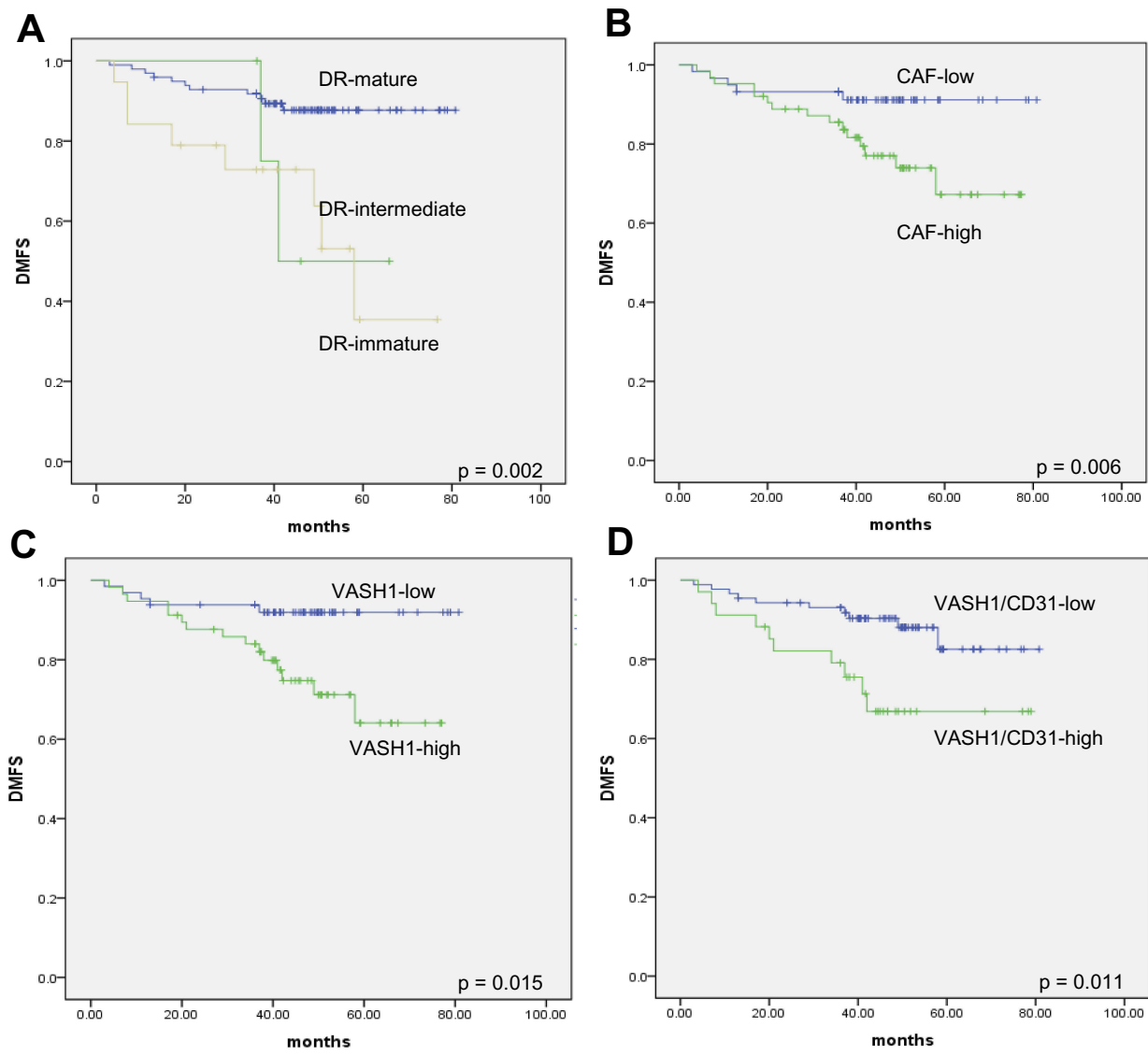

Figure 3 Kaplan-Meier curves of distant metastasis-free survival of patients with cervical squamous cell carcinoma. (A) DR; (B) CAFs; (C) VASHI-positive ratio; (D) VASHI/CD3I ratio.

categorization was an independent factor for the prediction of survival in the current study.

Few studies have focused on the morphological variations of the stroma pattern in CxSCC histopathology. The DR categorization method, in this study, was based on the reaction components, and the fibrotic environment in front of tumors is dynamic. Additionally, both mature and immature stroma are histological features that represent the status of ECM remodeling that occurs when the tumor cell has the potential to disseminate or metastasize.

The molecular basis of the association between poor outcomes and unfavorable DR categories has not been clarified in patients with CxSCC. Previous studies have revealed that immature stroma are associated with higher activity of tumor budding. ${ }^{11,22}$ The tumor dedifferentiation phenotypes at the invasive border are a morphologic feature of epithelialmesenchymal transition (EMT) ${ }^{23,24}$ which is a vital molecular mechanism that allows tumor cells to obtain the ability to invade, survive, and metastasize. ${ }^{23}$ Therefore, CAFs in tumors with immature stroma may regulate the tumor microenvironment through releasing various types of growth factors, cytokines and chemokines that enhanced EMT and subsequently drive metastasis. CAFs accumulate in the tumor microenvironment, they are activated by the surrounding growth factors and cytokines, such as TGF- $\beta$, MCP1, plateletderived growth factor, fibroblast growth factor, and secreted proteases. ${ }^{8,25}$ Following activation, CAFs secreted growth factors that promote tumorigenesis. ${ }^{26}$ Additionally, CAFs also produce pro-inflammatory factors that activate NFKB signaling to promote tumorigenesis. ${ }^{27}$ In contrast, the DR correlated with the immune microenvironment; that is, previous studies revealed that immature stroma correlated with less angiogenesis ${ }^{28}$ and could be a barrier to immune cell infiltration. ${ }^{11,12,28}$ It is recognized that CAFs and ECM generated a physical barrier to immune cell infiltration into tumors. ${ }^{29}$

CAFs enhance a "mesenchymal-like" phenotype and promote metastasis of neoplastic epithelial cells, whereas the normal fibroblasts confer an "epithelial-like" phenotype and suppress metastasis. ${ }^{30}$ It indicates that CAFs are a totally distinct cell type from normal fibroblasts that have potent effects on tumorigenesis. ${ }^{31}$ Confirmed with earlier studies, our current data demonstrated that CAFs and myxoid stroma were 
unfavorable predictors of DMFS in CXSCC patients, while fibroblastic stroma was a favorable predictor of DMFS. Currently, there are no effective histological methods to distinguish heterogeneous populations of CAFs; however, DR categorization may be used as a histopathological method that helps verify the heterogeneity of CAFs, and this method may be utilized in CxSCC treatment.

Nestin is an intermediate filament protein and expressed in newly formed vessels. Nestin also expresses in pericytes around microvessels. ${ }^{7}$ Pericytes are essential in maintaining the stability of the vessel because they support the vascular walls. ${ }^{32}$ In this present study, MVD was significantly higher in T1b group than that in T1a group; however, the nestin-positive ratio was significantly lower in N1 group than that in N0 group. Further, intratumoral microvessels that are not surrounded by pericytes have higher permeability and lower vulnerability.

Our study had several limitations. Firstly, it is a singlecenter retrospective study with small cohort size and, therefore, the statistical power was limited. Secondly, we only evaluated stromal features such as DR, CAF, and MVD in $\mathrm{CxSCC}$ despite the fact that those stromal features may also be critical prognostic factors in surgically resected adenocarcinomas. Therefore, more studies should include cervical cancer with more histologic types. Finally, our study focused on identifying the top predictor for distant metastasis; however, the generalizability of current results is unclear. Therefore, further studies for validation are required.

VASH1 is an anti-angiogenic factor that was derived from endothelial cells. ${ }^{5,6}$ Our results showed that the VASH1positive ratio and the VASH1/CD31 ratio were directly correlated with unfavorable DMFS. Therefore, our data suggest that VASH1 may be a value predictor of anti-angiogenesis therapy for patients with CXSCC. However, further studies in vitro are needed to confirm these speculations.

\section{Conclusion}

Our data highlights the prognostic value of histopathological examinations of stromal features for distant metastases. By reproducible multivariate model risk analysis, our results provided a robust model for survival stratification. Furthermore, these data provide evidence for the production of the novel histopathological predictor that improves DMFS prediction in CXSCC patients and may eventually improve the standard histological practices.

\section{Acknowledgment}

The authors would like to thank Mr. Xianliang Sha for providing technical assistance.

\section{Disclosure}

The authors report no conflicts of interest in this work.

\section{References}

1. Lee MY, Chou CY, Tang MJ, Shen MR. Epithelial-mesenchymal transition in cervical cancer: correlation with tumor progression, epidermal growth factor receptor overexpression, and snail up-regulation. Clin Cancer Res. 2008;14(15):4743-4750. doi:10.1158/1078-0432.CCR08-0234

2. Marth C, Landoni F, Mahner S, et al. Cervical cancer: ESMO clinical practice guidelines for diagnosis, treatment and follow-up. Ann Oncol. 2018;29(Supplement_4):iv262. doi:10.1093/annonc/mdy160

3. Deligeoroglou E, Giannouli A, Athanasopoulos N, et al. HPV infection: immunological aspects and their utility in future therapy. Infect Dis Obstet Gynecol. 2013;2013:540850. doi:10.1155/2013/540850

4. Uzzan B, Nicolas P, Cucherat M, Perret GY. Microvessel density as a prognostic factor in women with breast cancer: a systematic review of the literature and meta-analysis. Cancer Res. 2004;64 (9):2941-2955. doi:10.1158/0008-5472.CAN-03-1957

5. Sato Y. The vasohibin family: a novel family for angiogenesis regulation. J Biochem. 2013;153(1):5-11. doi:10.1093/jb/mvs128

6. Tamaki K, Moriya T, Sato Y, et al. Vasohibin-1 in human breast carcinoma: a potential negative feedback regulator of angiogenesis. Cancer Sci. 2009;100(1):88-94. doi:10.1111/cas.2009.100.issue-1

7. Matsuda Y, Hagio M, Ishiwata T. Nestin: a novel angiogenesis marker and possible target for tumor angiogenesis. World J Gastroenterol. 2013;19(1):42-48. doi:10.3748/wjg.v19.i1.42

8. Kalluri R, Zeisberg M. Fibroblasts in cancer. Nat Rev Cancer. 2006;6 (5):392-401. doi:10.1038/nrc1877

9. Ohlund D, Elyada E, Tuveson D. Fibroblast heterogeneity in the cancer wound. J Exp Med. 2014;211(8):1503-1523. doi:10.1084/ jem.20140692

10. Megumi K, Ishigami S, Uchikado Y, et al. Clinicopathological significance of BMP7 expression in esophageal squamous cell carcinoma. Ann Surg Oncol. 2012;19(6):2066-2071. doi:10.1245/ s10434-011-2024-5

11. Ueno H, Jones AM, Wilkinson KH, Jass JR, Talbot IC. Histological categorisation of fibrotic cancer stroma in advanced rectal cancer. Gut. 2004;53(4):581-586. doi:10.1136/gut.2003.028365

12. Ueno H, Jones A, Jass JR, Talbot IC. Clinicopathological significance of the 'keloid-like' collagen and myxoid stroma in advanced rectal cancer. Histopathology. 2002;40(4):327-334. doi:10.1046/j.13652559.2002.01376.X

13. Ao T, Kajiwara Y, Yonemura K, et al. Prognostic significance of histological categorization of desmoplastic reaction in colorectal liver metastases. Virchows Arch. 2019;475(3):341-348. doi:10.1007/ s00428-019-02580-2

14. Ueno $\mathrm{H}$, Shinto E, Hashiguchi $\mathrm{Y}$, et al. In rectal cancer, the type of desmoplastic response after preoperative chemoradiotherapy is associated with prognosis. Virchows Arch. 2015;466(6):655-663. doi:10.1007/s00428-015-1756-1

15. Vered M, Dobriyan A, Dayan D, et al. Tumor-host histopathologic variables, stromal myofibroblasts and risk score, are significantly associated with recurrent disease in tongue cancer. Cancer Sci. 2010;101(1):274-280. doi:10.1111/cas.2009.101.issue-1

16. Jesinghaus M, Boxberg M, Konukiewitz B, et al. A novel grading system based on tumor budding and cell nest size is a strong predictor of patient outcome in esophageal squamous cell carcinoma. Am J Surg Pathol. 2017;41(8):1112-1120. doi:10.1097/PAS.0000000000000865

17. Boxberg M, Jesinghaus M, Dorfner C, et al. Tumour budding activity and cell nest size determine patient outcome in oral squamous cell carcinoma: proposal for an adjusted grading system. Histopathology. 2017;70(7):1125-1137. doi:10.1111/his.2017.70.issue-7 
18. Weichert W, Kossakowski C, Harms A, et al. Proposal of a prognostically relevant grading scheme for pulmonary squamous cell carcinoma. Eur Respir J. 2016;47(3):938-946. doi:10.1183/ 13993003.00937-2015

19. Armstrong RA. When to use the Bonferroni correction. Ophthalmic Physiol Opt. 2014;34(5):502-508. doi:10.1111/opo.12131

20. Cao L, Wen H, Feng Z, Han X, Wu X. Distinctive clinicopathologic characteristics and prognosis for different histologic subtypes of early cervical cancer. Int J Gynecol Cancer. 2019;29:1244-1251. doi:10.1136/ijgc-2019-000556

21. Ueno H, Shinto E, Kajiwara Y, et al. Prognostic impact of histological categorisation of epithelial-mesenchymal transition in colorectal cancer. $B r J$ Cancer. 2014;111(11):2082-2090. doi:10.1038/bjc.2014.509

22. Ueno H, Kanemitsu Y, Sekine S, et al. Desmoplastic pattern at the tumor front defines poor-prognosis subtypes of colorectal cancer. Am J Surg Pathol. 2017;41(11):1506-1512. doi:10.1097/ PAS.0000000000000946

23. Hanahan D, Weinberg RA. Hallmarks of cancer: the next generation. Cell. 2011;144(5):646-674. doi:10.1016/j.cell.2011.02.013

24. Hainaut P, Plymoth A. Targeting the hallmarks of cancer: towards a rational approach to next-generation cancer therapy. Curr Opin Oncol. 2013;25(1):50-51. doi:10.1097/CCO.0b013e32835b651e

25. Marsh T, Pietras K, McAllister SS. Fibroblasts as architects of cancer pathogenesis. Biochim Biophys Acta. 2013;1832(7):1070-1078. doi:10.1016/j.bbadis.2012.10.013
26. Fukumura D, Xavier R, Sugiura T, et al. Tumor induction of VEGF promoter activity in stromal cells. Cell. 1998;94(6):715-725. doi:10.1016/S0092-8674(00)81731-6

27. Erez N, Truitt M, Olson P, Arron ST, Hanahan D. Cancer-associated fibroblasts are activated in incipient neoplasia to orchestrate tumor-promoting inflammation in an NF-kappaB-dependent manner. Cancer Cell. 2010;17(2):135-147. doi:10.1016/j.ccr.2009.12.041

28. Ueno H, Shinto E, Shimazaki H, et al. Histologic categorization of desmoplastic reaction: its relevance to the colorectal cancer microenvironment and prognosis. Ann Surg Oncol. 2015;22(5):1504-1512. doi:10.1245/s10434-014-4149-9

29. Kalluri R. The biology and function of fibroblasts in cancer. Nat Rev Cancer. 2016;16(9):582-598. doi:10.1038/nrc.2016.73

30. Dumont N, Liu B, Defilippis RA, et al. Breast fibroblasts modulate early dissemination, tumorigenesis, and metastasis through alteration of extracellular matrix characteristics. Neoplasia. 2013;15 (3):249-262. doi:10.1593/neo. 121950

31. van Zijl F, Mair M, Csiszar A, et al. Hepatic tumor-stroma crosstalk guides epithelial to mesenchymal transition at the tumor edge. Oncogene. 2009;28(45):4022-4033. doi:10.1038/onc.2009.253

32. Bonkowski D, Katyshev V, Balabanov RD, Borisov A, Dore-Duffy P. The CNS microvascular pericyte: pericyte-astrocyte crosstalk in the regulation of tissue survival. Fluids Barriers CNS. 2011;8(1):8. doi: $10.1186 / 2045-8118-8-8$

\section{Publish your work in this journal}

Cancer Management and Research is an international, peer-reviewed open access journal focusing on cancer research and the optimal use of preventative and integrated treatment interventions to achieve improved outcomes, enhanced survival and quality of life for the cancer patient.
The manuscript management system is completely online and includes a very quick and fair peer-review system, which is all easy to use Visit http://www.dovepress.com/testimonials.php to read real quotes from published authors. 